Does high optimism protect against the inter-generational transmission of high BMI? The Cardiovascular Risk in Young Finns Study

Serlachius, Anna

2017-09

Serlachius , A, Pulkki-Råback , L , Juonala , M , Sabin , M , Lehtimäki , T , Raitakari , O \& Elovainio, M 2017 , ' Does high optimism protect against the inter-generational transmission of high BMI? The Cardiovascular Risk in Young Finns Study ', Journal of Psychosomatic Research, vol. 100 , pp. 61-64 . https://doi.org/10.1016/j.jpsychores.2017.07.006

http://hdl.handle.net/10138/299536

https://doi.org/10.1016/j.jpsychores.2017.07.006

publishedVersion

Downloaded from Helda, University of Helsinki institutional repository.

This is an electronic reprint of the original article.

This reprint may differ from the original in pagination and typographic detail.

Please cite the original version. 
Short communication

\title{
Does high optimism protect against the inter-generational transmission of high BMI? The Cardiovascular Risk in Young Finns Study
}

\author{
Anna Serlachius $^{\mathrm{a}, \mathrm{b}, *}$, Laura Pulkki-Råback ${ }^{\mathrm{b}, \mathrm{c}}$, Markus Juonala ${ }^{\mathrm{d}, \mathrm{e}}$, Matthew Sabin ${ }^{\mathrm{f}, \mathrm{g}}$, \\ Terho Lehtimäki ${ }^{\mathrm{h}, \mathrm{i}}$, Olli Raitakarij ${ }^{\mathrm{j}, \mathrm{k}}$, Marko Elovainio ${ }^{\mathrm{b}, \mathrm{l}}$ \\ a The Department of Psychological Medicine, The University of Auckland, New Zealand \\ b Department of Psychology and Logopedics, The University of Helsinki, Finland \\ c The Collegium for Advanced Studies, University of Helsinki, Finland \\ d Department of Medicine, University of Turku, Finland \\ e The Division of Medicine, Turku University Hospital, Turku, Finland \\ ${ }^{f}$ The Department of Paediatrics, University of Melbourne, Australia \\ ${ }^{g}$ Murdoch Childrens Research Institute, Royal Children's Hospital, Melbourne, Australia \\ h The Department of Clinical Chemistry, Fimlab Laboratories, Tampere, Finland \\ ${ }^{i}$ Faculty of Medicine and Life Sciences, University of Tampere, Tampere, Finland \\ $\mathrm{j}$ The Department of Clinical Physiology and Nuclear Medicine, Turku University Hospital, Turku, Finland \\ ${ }^{\mathrm{k}}$ Research Centre of Applied and Preventive Cardiovascular Medicine, University of Turku, Turku, Finland \\ ${ }^{1}$ Institute for Health and Welfare, Helsinki, Finland
}

\section{A R T I C L E I N F O}

\section{Keywords:}

Optimism

BMI

Obesity

\begin{abstract}
A B S T R A C T
Objective: The transmission of overweight from one generation to the next is well established, however little is known about what psychosocial factors may protect against this familial risk. The aim of this study was to examine whether optimism plays a role in the intergenerational transmission of obesity.

Methods: Our sample included 1043 participants from the prospective Cardiovascular Risk in Young FINNS Study. Optimism was measured in early adulthood (2001) when the cohort was aged 24-39 years. BMI was measured in 2001 (baseline) and 2012 when they were aged 35-50 years. Parental BMI was measured in 1980. Hierarchical linear regression and logistic regression were used to examine the association between optimism and future BMI/obesity, and whether an interaction existed between optimism and parental BMI when predicting BMI/obesity 11 years later.

Results: High optimism in young adulthood demonstrated a negative relationship with high BMI in mid-adulthood, but only in women $(\beta=-0.127, p=0.001)$. The optimism $\times$ maternal BMI interaction term was a significant predictor of future BMI in women $(\beta=-0.588, p=0.036)$. The logistic regression results confirmed that high optimism predicted reduced obesity in women ( OR $=0.68,95 \% \mathrm{CI}, 0.55-0.86$ ), however the optimism $\times$ maternal obesity interaction term was not a significant predictor (OR $=0.50,95 \% \mathrm{CI}, 0.10-2.48$ ). Conclusions: Our findings supported our hypothesis that high optimism mitigated the intergenerational transmission of high BMI, but only in women. These findings also provided evidence that positive psychosocial factors such as optimism are associated with long-term protective effects on BMI in women.
\end{abstract}

\section{Introduction}

Parental overweight and obesity are well-known risk factors for offspring obesity, yet few studies have examined protective psychosocial factors that may mitigate the spread of obesity from one generation to the next. Psychosocial factors which are likely implicated in the intergenerational transmission of overweight include childhood socioeconomic status (SES), depression, psychosocial stress, and social support [1-4]. Another protective psychosocial factor, which may play a similar role, is optimism, generally defined as having a positive outlook about the future [5].

Optimism has shown to positively associate with a healthy diet and a low body mass index (BMI) in a population-based study of young adults [6], and with healthy behaviours including increased physical activity and eating a healthier diet in elderly men [7]. Another study found that in a cohort of middle-aged adults, optimism was associated with a healthier lipid profile, mediated partly by health behaviours

\footnotetext{
* Corresponding author at: Department of Psychological Medicine, The University of Auckland, Private Bag 92019, Victoria Street West, Auckland 1142, New Zealand.

E-mail address: a.serlachius@auckland.ac.nz (A. Serlachius).
} 
such as diet as well as BMI [8]. Despite emerging research assessing the impact of optimism on BMI and weight gain, research examining optimism in the context of the intergenerational transmission of obesity is lacking.

Boehm and Kubzansky [9] recently developed a model to explain the association between positive psychological factors (including optimism) and physical health. The model suggests that the association between positive psychological wellbeing and cardiovascular disease is mediated by restorative processes, which help to buffer against ill health (e.g. eating a healthy diet), as well as by the absence of deteriorative processes that negatively affect health (e.g. smoking). These restorative and deteriorative processes include health behaviours as well as biological functions (e.g. inflammation or cholesterol).

In line with this model, we wanted to explore whether optimism buffers against the familial transmission of high BMI. Using data from the Cardiovascular Risk in Young Finns Study (Young Finns Study), a prospective study design initiated 37 years ago; we examined whether: [1] optimism in young adulthood predicts BMI in mid-adulthood, and [2] optimism interacts with parental BMI to mitigate the intergenerational transmission of high BMI.

\section{Methods}

The Young Finns Study is a prospective cohort study initiated in 1980 to examine cardiovascular risk factors in a randomly selected cohort of 3596 Finnish children aged 3-18 years old (see Raitakari et al.) [10]. The current study included an analytic sample of 1043 participants with no missing data on key variables: parental BMI, parental socioeconomic status, BMI in adulthood, and optimism in adulthood. Participants provided written informed consent and the study was approved by the ethics committees of the participating universities.

Optimism was measured in 2001 when participants were 24-39 years, using the Life Orientation Test-Revised [11,12], a self-report questionnaire that measures positive expectancies about the future. The questionnaire has six items of which three are worded positively and three negatively. Items are rated on a 5 -point Likert scale $(0=$ not at all and $4=$ very much so). The score can range from 0 to 24 (whereby the negative items are reversed scored), so that a high score represents high optimism. The scale demonstrates good validity and reliability [12]. In the present sample, the Cronbach alpha was $\alpha=0.78$.

BMI was measured both in 2001 (to adjust for baseline BMI) as well as in 2012 when participants were aged $35-50$ years. Weight and height were measured by trained staff. BMI was calculated with the formula: BMI $=$ weight $(\mathrm{kg}) /[\text { height }(\mathrm{m})]^{2}$. Maternal and paternal height and weight were assessed using self-report questionnaires in 1980 and parental BMI was calculated using the above formula.

Parental occupation was assessed with self-report questionnaires in 1980. Parental occupation was coded according to the categories used by the Central Statistical Office of Finland from 1 to $3(1=$ manual, 2 = lower non-manual, $3=$ upper non-manual) and were used as a proxy of SES.

\subsection{Statistical analyses}

After conducting attrition analyses, we used hierarchical regression to examine the association between parental BMI (maternal and paternal separately) and BMI in the cohort in mid-adulthood, with all models adjusted for age and parental SES (Model 1). We then sequentially adjusted for optimism (Model 2) and the interaction term between maternal BMI and optimism, and paternal BMI and optimism (Model 3). In our supplementary analysis we also adjusted for BMI at baseline (measured at the same time as optimism in 2001), in order to examine the direction of the relationship between optimism and BMI. All continuous variables were standardized (Mean $=0, S D=1$ ). Due to the significant interaction between optimism and sex $(p=0.033)$ when predicting BMI in 2012, we separated all the analyses by sex. We repeated the main analyses using logistic regression, with a binary dependent variable (BMI > 30). We used odds ratios (ORs) with $95 \%$ confidence intervals (CI) to examine how the odds of being obese in adulthood were associated with parental obesity at baseline, and adjusted for the above covariates. Lastly, in order to visualize the conditional effect of parental BMI on BMI in mid-adulthood at low ( -1 SD from mean), medium (mean) and high levels ( +1 SD from mean) of optimism, we used a simple slopes analysis using the PROCESS macro [13]. All the analyses were performed using SPSS [23].

\section{Results}

Attrition analyses showed that participants in the analytic sample had higher optimism ( $p<0.001$, Cohen's $d=0.18$ ) as well as lower adulthood BMI ( $\mathrm{p}=0.013$, Cohen's $\mathrm{d}=0.11$ ) and baseline BMI measured in 2001 ( $p=0.003$, Cohen's $d=0.12$ ), compared to participants from the broader sample. There were no differences in maternal BMI ( $p=0.265$, Cohen's $d=0.04$ ) or paternal BMI ( $p=0.997$, Cohen's $\mathrm{d}=0.00$ ). The parents from the analytic sample were also more likely to have a higher SES ( $\mathrm{p}<0.001)$.

Out of the 1043 participants, 645 were women (61.8\%). Average BMI for women in adulthood was $25.9 \mathrm{~kg} / \mathrm{m}^{2}$ ( \pm 5.3 ), out of which 125 had a BMI $>30$. Average BMI for men in adulthood was $26.6 \mathrm{~kg} /$ $\mathrm{m}^{2}( \pm 4.2)$, and 77 had a BMI $>30$. Mean optimism in adulthood was 17.5 ( \pm 3.7) for women and 17.2 ( \pm 3.8$)$ for men. The cohort's mothers' mean BMI was $24 \mathrm{~kg} / \mathrm{m}^{2}$ ( \pm 3.7$)$, out of which 70 had a BMI $>30$ at baseline. The fathers' BMI was $25.5 \mathrm{~kg} / \mathrm{m}^{2}( \pm 3.0)$ and 85 had a BMI > 30. Parental SES was grouped as low $(\mathrm{n}=259,24.8 \%)$, medium ( $n=634,60.8 \%)$, and high $(n=150,14.4 \%)$.

Due to the significant sex $\times$ optimism interaction term $(\mathrm{p}=0.033)$ when predicting BMI in 2012, the following analyses were separated by sex. In the regression models, high optimism in young adulthood demonstrated a negative relationship with high BMI in mid-adulthood, but only in women ( $\beta=-0.127, p=0.001$, Table 1$)$. Optimism was not a significant predictor of future BMI in men $(\beta=-0.017$, $\mathrm{p}=0.728$ ), therefore the results focus on women. Maternal BMI was positively associated with BMI in mid-adulthood across all models. Only the maternal (not the paternal) BMI $\times$ optimism interaction term was significant in predicting BMI in $2012(\beta=-0.588, p=0.036$ versus $\beta=-0.148, p=0.676)$. Once we adjusted for the maternal BMI $\times$ optimism interaction term, optimism was no longer a significant predictor of BMI in women $(\beta=0.503, \mathrm{p}=0.180$ ), indicating moderation.

The simple slopes analysis confirmed these results, and indicated that low optimism in young adulthood increased the risk of having a high BMI if the mother had a high BMI (Fig. 1). As a supplementary analysis (see Supplementary Table 1), we also repeated the linear regression analysis (Models 1-3) and adjusted for BMI at baseline (measured in 2001), in order to examine the temporal relationship between optimism and BMI in women. Optimism remained a significant predictor of BMI in 2012, even after adjusting for baseline BMI ( $\beta=-0.068, p=0.001)$. However, the interaction between maternal BMI and optimism was no longer a predictor of BMI in 2012 $(\beta=-0.048, p=0.755)$.

Lastly, the logistic regression also found that high optimism predicted reduced future obesity in women but not men (OR $=0.68,95 \%$ CI, $0.55-0.86, \mathrm{p}=0.001$ compared to OR $=0.98,95 \%$ CI, $0.75-1.29$, $\mathrm{p}=0.947$, see Supplementary Table 2). However, the interaction term between optimism $\times$ maternal obesity was not a significant predictor of obesity in women (OR $=0.50,95 \% \mathrm{CI}, 0.10-2.48, \mathrm{p}=0.396)$.

\section{Discussion}

To our knowledge, this is the first study to demonstrate the protective effects of high optimism in the intergenerational transmission of high BMI, thereby also adding to the literature on positive psychological factors and 
Table 1

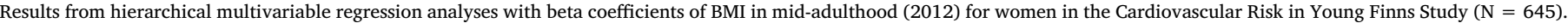
Model 3 adjusts for the interaction term between maternal BMI and optimism and the interaction term between paternal BMI and optimism.

\begin{tabular}{|c|c|c|c|}
\hline Predictors & $\begin{array}{l}\text { Model } 1 \\
\beta^{\text {a }} \text { (p-Value) }\end{array}$ & $\begin{array}{l}\text { Model } 2 \\
\beta^{\text {a }}(\mathrm{p}-\text { Value })\end{array}$ & $\begin{array}{l}\text { Model } 3 \\
\beta^{\text {a }} \text { (p-Value) }\end{array}$ \\
\hline Age & $0.027(\mathrm{p}=0.471)$ & $0.038(\mathrm{p}=0.316)$ & $0.031(\mathrm{p}=0.405)$ \\
\hline Maternal BMI kg/m² (1980) & $0.226(\mathrm{p}<0.001)$ & $0.219(\mathrm{p}<0.001)$ & $0.565(\mathrm{p}=\mathbf{0 . 0 0 1})$ \\
\hline Paternal BMI kg/m² (1980) & $0.222(\mathrm{p}<0.001)$ & $0.212(\mathrm{p}<0.001)$ & $0.291(\mathrm{p}=0.102)$ \\
\hline Parental occupational status (Low) & Reference & Reference & Reference \\
\hline Parental occupational status (Medium) & $0.152(\mathrm{p}<0.001)$ & $0.136(p=0.002)$ & $0.143(p=0.001)$ \\
\hline Parental occupational status (High) & $0.078(\mathrm{p}=0.067)$ & $0.059(\mathrm{p}=0.164)$ & $0.066(\mathrm{p}=0.123)$ \\
\hline Optimism (2001) & & $-0.127(\mathbf{p}=\mathbf{0 . 0 0 1})$ & $0.503(\mathrm{p}=0.180)$ \\
\hline Maternal BMI $\times$ optimism & & & $-0.588(p=0.036)$ \\
\hline Paternal BMI $\times$ optimism & & & $-0.148(\mathrm{p}=0.676)$ \\
\hline Change in $\mathrm{R}^{2}$ & & & $0.006(\mathrm{p}=0.087)$ \\
\hline
\end{tabular}

The bold values are significant at $\mathrm{p}<0.05$. BMI $=$ Body Mass Index.

${ }^{\text {a }}$ Standardized beta coefficients.

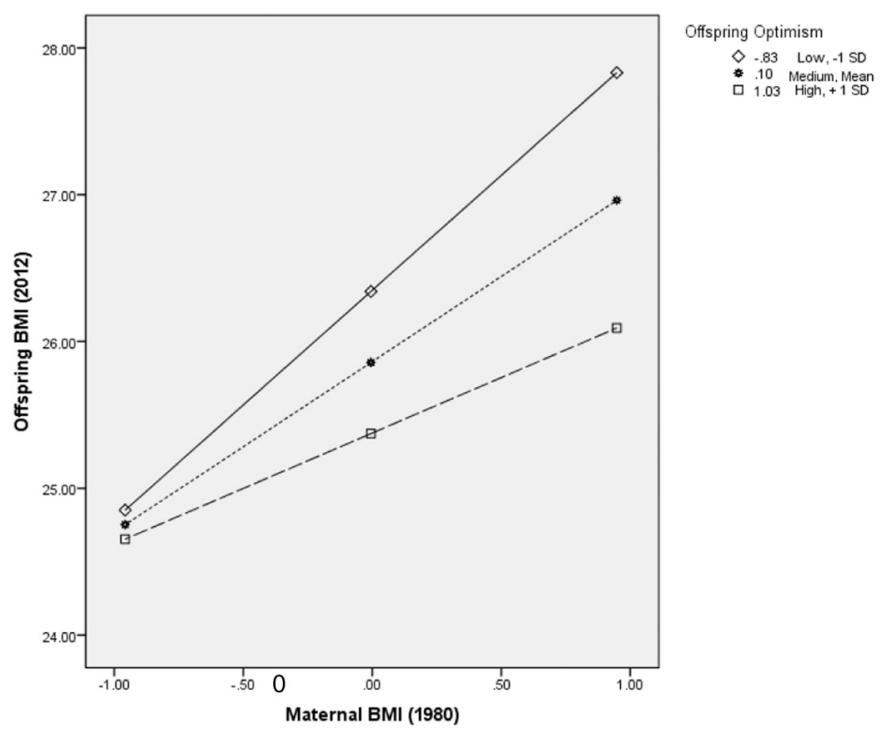

Fig. 1. The relation between maternal BMI and BMI in mid-adulthood (2012) at low $(\mathrm{p}<0.001)$, medium $(\mathrm{p}<0.001)$ and high $(\mathrm{p}=0.014)$ levels of optimism in women from the Cardiovascular Risk in Young Finns Study $(n=645)$.

health outcomes [9]. Another novel finding was that optimism was related to change in BMI over time, with the supplementary analysis (where we adjusted for baseline BMI) suggesting that high optimism is driving low BMI. Both of these findings were only significant in women. Although our main results demonstrated that optimism in young adulthood interacts with parental BMI to moderate the intergenerational transmission of high BMI, when we adjusted for baseline BMI, this interaction was no longer significant. This seems to suggest that perhaps instead of preventing the growth of high BMI, high optimism may prevent initially high levels of BMI in women with high maternal BMI.

As we did not set out to test possible mechanisms for the protective effect of high optimism, we can only speculate on the reasons why high optimism in women seems to be protecting against future weight gain. High optimism has been linked to protective health behaviours, suggesting that optimistic people may be more likely to adopt and maintain healthy behaviours $[14,15]$. Another possible pathway between high optimism and a healthier BMI is that optimism may protect against psychosocial stress, which has been associated with long-term weight gain and increased central adiposity [16,17].

It was unexpected that optimism did not independently predict future weight gain in men. The literature on sex-specific effects of protective psychosocial factors, such as optimism, on health outcomes is scarce. However, other studies have found that maladaptive psychosocial factors such as stress can have sex-specific effects on health outcomes including
BMI and Type 2 diabetes [18,19]. One possibility for the sex-specific outcomes in our study could be that having a positive outlook has a stronger association with certain health behaviours that protect against weight gain in women compared to men. Previous studies have found that low mood and psychological distress was associated with different health behaviours in men and women, including poor dietary behaviours and lack of exercise in women $[20,21]$. Alternatively, perhaps optimism protects women more than men against chronic psychosocial stress and its impact on health, for which women may be more vulnerable [22], and which may exacerbate weight gain [17].

Strengths of our study include a longitudinal design that allowed us to examine the association between optimism and BMI 11 years later. The cohort is also unique in that we have data from two generations, allowing us to investigate intergenerational phenomena. Limitations include a relatively homogenous population, self-reported parental BMI, and attrition over the more than three decades; however, it has not been significant enough to bias findings $[23,24]$. Lastly, we only measured optimism at one time point, making it difficult to rule out that optimism did not change during the follow-up period. However, optimism is generally seen as a stable trait [5], with approximately $25 \%$ of optimism thought to be due to genetic factors [25].

In summary, our results suggest that high optimism protects against high BMI in women over time, and that high optimism moderates the intergenerational transmission of BMI in women. The temporal nature of the latter relationship warrants further investigation. Our findings are consistent with emerging evidence suggesting that positive psychosocial factors, both in childhood and in adulthood, are associated with long-term protective effects on metabolic functioning $[26,27]$.

\section{Funding sources}

The Young Finns Study has been financially supported by the Academy of Finland: grants 286284, 134309 (Eye), 126925, 121584, 124282, 129378 (Salve), 117787 (Gendi), and 41071 (Skidi); the Social Insurance Institution of Finland; Competitive State Research Financing of the Expert Responsibility area of Kuopio, Tampere and Turku University Hospitals (grant X51001); Juho Vainio Foundation; Paavo Nurmi Foundation; Finnish Foundation for Cardiovascular Research; Finnish Cultural Foundation; Tampere Tuberculosis Foundation; Emil Aaltonen Foundation; Yrjö Jahnsson Foundation; Signe and Ane Gyllenberg Foundation; and Diabetes Research Foundation of Finnish Diabetes Association.

\section{Conflict of interest}

The authors have no potential conflicts of interest to disclose. The authors have no financial relationships relevant to this article to disclose. 


\section{Acknowledgements}

We greatly acknowledge Irina Lisinen, Johanna Ikonen and Ville Aalto for assistance in managing the Cardiovascular Risk in Young Finns dataset.

\section{Appendix A. Supplementary data}

Supplementary data to this article can be found online at http://dx. doi.org/10.1016/j.jpsychores.2017.07.006.

\section{References}

[1] A.M. Lampard, R.L. Franckle, K.K. Davison, Maternal depression and childhood obesity: a systematic review, Prev. Med. 59 (2014) 60-67.

[2] J. Costa-Font, J. Gil, Intergenerational and socioeconomic gradients of child obesity, Soc. Sci. Med. 93 (2013) 29-37.

[3] C. Gundersen, D. Mahatmya, S. Garasky, B. Lohman, Linking psychosocial stressors and childhood obesity, Obes. Rev. 12 (5) (2011) e54-e63.

[4] A. Serlachius, M. Elovainio, M. Juonala, S. Shea, M. Sabin, T. Lehtimäki, et al., High perceived social support protects against the intergenerational transmission of obesity: the Cardiovascular Risk in Young Finns Study, Prev. Med. 90 (2016) 79-85.

[5] C.S. Carver, M.F. Scheier, S.C. Segerstrom, Optimism, Clin. Psychol. Rev. 30 (2010) 879-889.

[6] H. Kelloniemi, E. Ekb, J. Laitinen, Optimism, dietary habits, body mass index and smoking among young Finnish adults, Appetite 45 (2005) 169-176.

[7] E.J. Giltay, J.M. Geleijnse, F.G. Zitman, B. Buijsse, D. Kromhout, Lifestyle and dietary correlates of dispositional optimism in men: the Zutphen Elderly Study, J. Psychosom. Res. 63 (5) (2007) 483-490.

[8] J.K. Boehm, D.R. Williams, E.B. Rimm, C. Ryff, L.D. Kubzansky, Relation between optimism and lipids in midlife, Am. J. Cardiol. 111 (10) (2013) 1425-1431.

[9] J.K. Boehm, L.D. Kubzansky, The heart's content: the association between positive psychological well-being and cardiovascular health, Psychol. Bull. 138 (4) (2012) 655-691.

[10] O.T. Raitakari, M. Juonala, T. Rönnemaa, L. Keltikangas-Järvinen, L. Räsänen, M. Pietikäinen, et al., Cohort profile: the Cardiovascular Risk in Young Finns Study, Int. J. Epidemiol. 37 (6) (2008) 1220-1226.

[11] M.F. Scheier, C.S. Carver, Optimism, coping, and health: assessment and implications of generalized outcome expectancies, Health Psychol. 4 (1985) 219-247.

[12] M.F. Scheier, C.S. Carver, M.W. Bridges, Distinguishing optimism from neuroticism (and trait anxiety, self-mastery, and self-esteem) - a reevaluation of the life orientation test, J. Pers. Soc. Psychol. 67 (6) (1994) 1063-1078.
[13] A.F. Hayes, Introduction to Mediation, Moderation, and Conditional Process Analysis: A Regression-based Approach, The Guilford Press, New York, 2013.

[14] A. Serlachius, L. Pulkki-Råback, M. Elovainio, M. Hintsanen, V. Mikkilä,

T.T. Laitinen, et al., Is dispositional optimism or dispositional pessimism predictive of ideal cardiovascular health? The Young Finns Study, Psychol. Health 30 (10) (2015) 1221-1239.

[15] A. Steptoe, C. Wright, S.R. Kunz-Ebrecht, S. Iliffe, Dispositional optimism and health behaviour in community-dwelling older people: associations with healthy ageing, Br. J. Health Psychol. 11 (1) (2006) 71-84.

[16] T. Van Strien, M. Rookus, G. Bergers, J. Frijters, P. Defares, Life events, emotional eating and change in body mass index, Int. J. Obes. 10 (1) (1985) 29-35.

[17] P. Björntorp, Do stress reactions cause abdominal obesity and comorbidities? Obes. Rev. 2 (2) (2001) 73-86.

[18] A.M. Heraclides, T. Chandola, D. R. Witte, E.J. Brunner, Work stress, obesity and the risk of type 2 diabetes: gender-specific bidirectional effect in the Whitehall II Study, Obesity 20 (2) (2012) 428-433.

[19] A. Heraclides, T. Chandola, D.R. Witte, E.J. Brunner, Psychosocial stress at work doubles the risk of type 2 diabetes in middle-aged women: evidence from the Whitehall II Study, Diabetes Care 32 (12) (2009) 2230-2235.

[20] A. Allgöwer, J. Wardle, A. Steptoe, Depressive symptoms, social support, and personal health behaviors in young men and women, Health Psychol. 20 (3) (2001) 223.

[21] S. Ezoe, K. Morimoto, Behavioral lifestyle and mental health status of Japanese factory workers, Prev. Med. 23 (1) (1994) 98-105.

[22] U. Lundberg, Stress hormones in health and illness: the roles of work and gender, Psychoneuroendocrinology 30 (10) (2005) 1017-1021.

[23] M. Juonala, J. Juhola, C.G. Magnussen, P. Wurtz, J.S. Viikari, R. Thomson, et al., Childhood environmental and genetic predictors of adulthood obesity: the cardiovascular risk in Young Finns Study, J. Clin. Endocrinol. Metab. 96 (9) (2011) (E1542-E9).

[24] L. Pulkki-Råback, M. Elovainio, C. Hakulinen, J. Lipsanen, M. Hintsanen, M. Jokela, et al., Cumulative effect of psychosocial factors in youth on ideal cardiovascular health in adulthood: the Cardiovascular Risk in Young Finns Study, Circulation 131 (2015) 245-253.

[25] R. Plomin, M.F. Scheier, C.S. Bergeman, N.L. Pedersen, J.R. Nesselroade, G.E. McClearn, Optimism, pessimism and mental health: a twin/adoption analysis, Personal. Individ. Differ. 13 (8) (1992) 921-930.

[26] M. Elovainio, L. Pulkki-Råback, C. Hakulinen, T. Lehtimäki, E. Jokinen, T. Rönnemaa, et al., Psychosocial environment in childhood and body mass index growth over 32 years, Prev. Med. 97 (2017) 50-55.

[27] L. Pulkki-Raback, M. Elovainio, C. Hakulinen, J. Lipsanen, L.D. Kubzansky, M. Hintsanen, ... M. Juonala, Positive Psychosocial Factors in Childhood Predicting Lower Risk for Adult Type 2 Diabetes: The Cardiovascular Risk in Young Finns Study, 1980-2012, Am. J. Prev. Med. 52 (6) (2017) e157-e164, http://dx.doi.org/ 10.1016/j.amepre.2017.01.042. 